\title{
Exploring Routley's Nuclear Meinongianism and Beyond
}

\author{
Naoya Fujikawa \\ Department of Language Sciences \\ Graduate School of Humanities, Tokyo Metropolitan University \\ fjnaoya@gmail.com
}

\begin{abstract}
Even though Richard Routley is known as a proponent of so-called nuclear Meinongianism (in his terminology, noneism), the details of his view in Exploring Meinong's Jungle and Beyond (1980) have not attracted much attention. In this paper, I critically examine two notable but less known features of Meinongianism in Routley (1980) which particularly concern a core claim of nuclear Meinongianism that so-called Characterization Principle (CP) is applicable to nuclear properties (in his terminology, characterizing properties) but not to extranuclear properties (in his terminology, noncharacterizing properties): The first one is his 'double determination' argument for restricting the class of properties to which $\mathrm{CP}$ is applicable; and the second is his expansion of the class of nuclear properties in terms of the supposition operator $s$. Firstly, I will show that the double determination argument fails, since, for some extranuclear properties, the argument fails to justify that $\mathrm{CP}$ is not applicable to them, and, moreover, the argument unintendedly shows that CP is not applicable to some nuclear properties. Secondly, based on the idea that the supposition operator, combined with any property $f$, forms the intensional property of being assumed to have $f$, I will give a formal treatment of the supposition operator within the framework of world semantics. We will see that this interpretation goes beyond Routley's original nuclear Meinongianism and assimilates his view with modal Meinongianism proposed by Priest $(2005,2016)$.
\end{abstract}

Australasian Journal of Logic (15:2) 2018, Article no. 2.1 


\section{Introduction: nuclear Meinongianism}

Meinongianism is the view that 'some objects do not exist, but we can generally refer to them, quantify over them, and make true claims about them' (Berto and Plebani, 2015, p. 100). There are several versions of Meinongianism, and the simplest and notoriously problematic one is naive Meinongianism. Naive Meiongianism is a version of Meinongianism which endorses the following naive Characterization Principle (henceforth, I use CP for Characterization Principle):

(1) naive CP: For any characterization (that is, any set of first-order properties), some (existent or nonexistent) object satisfies it (that is, it has all properties in the set).

Russell (1905) provided one of the most famous counterexamples to naive Meinongianism: The existent golden mountain. As Russell argued, from naive $\mathrm{CP}$, it follows that the existent golden mountain exists. Take the set of the following three properties: being existent, being golden and being a mountain. From naive CP, it follows that something has all of these properties, that is, the existent golden mountain exists. However, since, of course, there exists no golden mountain, it doesn't exist. A contradiction follows.

Meinongians, including Meinong himself, have proposed several solutions to this problem. One of such solutions is defended by nuclear Meinongianism (Jacquette, 2015; Parsons, 1980; Routley, 1980). Nuclear Meinongianism is based on the following two key ideas: (i) There are two different kinds of properties, that is, nuclear and extranuclear properties; and (ii) CP is applicable only to characterizations consisting of nuclear properties. Note that different authors use different terminologies for the two kinds of properties: Nuclear and extranuclear (Findlay, 1963; Parsons, 1980); constitutive and extraconstitutive (Jacquette, 2015); and characterizing and noncharacterizing properties/predicates (Routley, 1980). Even though this difference is not merely terminological, as long as our investigation in this paper concerns, we can regard these distinctions as basically the same one. ${ }^{1}$ To avoid the complexity, in what follows, we use the most popular one, that is, 'nuclear/extranuclear' terminology (even though different terminologies will appear in quotation). In sum, nuclear Meinongianism is the version of Meinongianism which endorses the following nuclear CP, instead of naive CP.

\footnotetext{
${ }^{1}$ For the difference between Parsons and Routley, see Routley (1980, p. 265, n 1).
} 
(2) nuclear CP: For any characterization which contains only nuclear properties (that is, any set of nuclear properties), some (existent or nonexistent) object satisfies it (that is, some object has all properties in the set). ${ }^{2}$

A nuclear Meinongian solution to the problem of the existent golden mountain goes as follows. ${ }^{3}$ According to nuclear Meinongianism, existence is not a nuclear property, but an extranuclear property. So, we can not apply $\mathrm{CP}$ to the set of the properties of being a mountain, being golden, and being existent. Therefore, it does not follow from nuclear CP that the existent golden mountain exists.

As we have already seen, a crucial assumption of nuclear Meinongianism is that there are two different kinds of properties, that is, nuclear and extranuclear properties. Then, first of all, which properties are nuclear and which ones are extranuclear? The following is a list of core examples of these two kinds of properties. ${ }^{4}$ On the one hand, nuclear properties are ordinary first-order properties like being blue, being tall, kicking Socrates, being kicked by Socrates, being golden, being a mountain, and so on. On the other hand, core examples of extranuclear properties include: Ontological properties like existence; modal properties like being (im)possible; intensional (and intentional) properties like being thought about by Russell; and technical properties like being (in)complete, being (in)consistent and so on. Secondly, how are these two kinds of properties distinguished? A standard exposition of the

\footnotetext{
${ }^{2}$ Routley formulates this as FCP, " $\square A(\xi x A(x))$, where $A(x)$ is a wff (containing just $x$ free) constructed in an allowable way from characterising predicate" (Routley, 1980, p. 342). Routley specified two allowable ways to construct complex predicates from nuclear predicates: Predicate negation and conjunction. Note also that Routley clearly distinguishes predicate negation and sentential negation and claims that the former generates a complex nuclear properties, but the latter doesn't.

${ }^{3}$ By incorporating the so-called watering-down principle, Parsons (1980) provides another, more complicated, solution to the problem. Routley also proposes a different complication concerning what properties the existent golden mountain has. See Section 3 and 4 of this paper.

${ }^{4}$ See Parsons (1980, p. 23); Routley (1980, p. 264-268). According to Routley, predicates to which $\mathrm{CP}$ is not applicable are sentential negation, higher-order (predicate or sentential) quantification, "or predicate defined in terms of such quantification, notably ontic and modal predicates such as $E$ and $\diamond$, logical predicates such as $\approx$ and $=$, and theoretical predicate such as 'determinate' and 'complete'" (Routley, 1980, pp. 260-261). $\mathrm{He}$ also holds that intensional properties are not characterizing properties. See Routley (1980, p. 266).
}

Australasian Journal of Logic (15:2) 2018 Article no. 2.1 
distinction is this: Nuclear properties are properties which constitute the nature of an object. On the other hand, extranuclear properties are properties which do not constitute the nature of an object and are "external" to it (cf. Routley, 1980, p. 856). Routley claims that the distinction corresponds to "the traditional distinction between essence-specifying [properties] and those that cannot be used in specifying the essence or nature of a thing" (Routley, 1980, p. 348). Moreover, some theorists claim that extranuclear properties are properties whose possession by an object is determined by its nuclear properties. According to Routley, extranuclear properties are "consequential, i.e. depend for their determination on the prior determination of lower order ones [=nuclear properties]" (Routley, 1980, p. 261). ${ }^{5}$

So far this has been a brief exposition of nuclear Meinongianism. Routley's Exploring Meinong's Jungle and Beyond (1980) is known as a detailed exposition and defense of nuclear Meinongianism. ${ }^{6}$ However, it is fair to say that not much attention has been paid to Routley's theory per se. Indeed, in the literature, Parsons (1980) is regarded as a representative of nuclear Meinongianism and it is often the case that Routley (1980) is just mentioned as one of the other attempts of developing nuclear Meinongianism and nothing more is stated on his view. ${ }^{7}$

However, Routley (1980) deserves more attention, since it has several remarkable features. First of all, Routley (1980) broadens the topics related with Meinongianism. For example, Routley's theory provides an extensive discussion about the importance of Meinongianism in mathematics and the theoretical sciences; it develops an interesting combination of Meinongianism and presentism; and it also contains historical considerations on Meinong's theory and other related theories like one by Thomas Reid. Secondly, Routley (1980) also tries to improve the nuclear Meinongian theory of nonexistent objects per se in some distinctive ways. Focusing on the latter, in this pa-

\footnotetext{
${ }^{5}$ Jacquette makes the same point:

The totalities of every intended object's Sosein of constitutive properties in turn provide the supervenience or ontic dependence base for the intended object's extraconstitutive ontic properties of existing or not existing, being possible or impossible, relevantly predicationally complete or incomplete, and the like. (Jacquette, 2015, p. xxx)
}

${ }^{6}$ Routley did not call his own view so. He named his theory of nonexistent objects noneism.

${ }^{7}$ For some exceptions, see, in particular, Rapaport (1984) and Paoletti (2013). 
per, I examine two such attempts which particularly concern a core claim of nuclear Meinongianism, that is, the restriction of CP's applicability. The first one is his "double determination" argument for restricting CP, according to which we can not apply CP to extranuclear properties, since whether an object has an extranuclear property or not is determined solely in terms of its nuclear properties; the second one is his extension of nuclear $\mathrm{CP}$ in terms of the supposition operator, $s$, which, combined with any extranuclear property $f$, creates a nuclear property of presenting itself as having $f$. These two attempts are worthy of special mention, since they are related to two well-known difficulties concerning nuclear Meinongianism. Firstly, it is often claimed that the nuclear Meinongian distinction between nuclear and extranuclear properties are $a d$ hoc and unmotivated. The double determination argument is a notable attempt to justify the distinction. Secondly, restricting $\mathrm{CP}$ only to nuclear properties allegedly results in the failure of making the distinction between an existent golden mountain and a golden mountain. By enlarging the class of nuclear properties in terms of the supposition operator, Routley tries to give a way to distinguish them.

This paper is structured as follows. In Section 2, I introduce Routley's double determination argument and critically examine it. We will see that the double determination argument fails for the following two reasons: Firstly, some extranuclear properties are such that whether an object has them is not determined solely by which nuclear properties it has; secondly, if the argument showed that CP is not applicable to extranuclear properties, it would also show that $\mathrm{CP}$ is not applicable to a certain range of nuclear properties. In section 3, I introduce the supposition operator $s$ and see how it expands the range of properties to which $\mathrm{CP}$ is applicable. I also examine how we can define the semantics of the indefinite description $\xi x A(x)$ by using the supposition operator. Finally, in Section 4, by interpreting the supposition operator as an intensional-predicate-forming operator, where $s(f)$ is interpreted as the property of being assumed to have $f$, I give a formal semantics of the supposition operator based on world semantics. We will see that this interpretation goes beyond Routley's original nuclear Meinongianism and assimilates his view with modal Meinongianism proposed by Priest (2005, 2016).

Australasian Journal of Logic (15:2) 2018 Article no. 2.1 


\section{Routley's double determination argument for restricting $\mathrm{CP}$}

There are several objections against nuclear Meinongianism. A typical criticism to it is that the restriction of CP adopted by nuclear Meinongianism is ad hoc and unmotivated. ${ }^{8}$ Priest says:

The problem for this line [that is, restricting the applicability of $\mathrm{CP}$ to a certain kind of properties] is to give a principled characterization of what constitutes a characterizing predicate and why. No one, as far as I am aware, has been able to do this. Certain classes of predicates can be circumscribed and deemed safe. But without an appropriate rationale, it is difficult to avoid the feeling that the class has been gerrymandered simply to avoid problems. ... As observed, it would appear to be the case that we can think about an object satisfying any set of conditions whatsoever. Phenomenologically, at least, there is absolutely no difference between contemplating an objet that has only officially characterizing properties ... and one that has some non-characterizing properties as well, say existence. Qua object of thought, each object seems to have all the properties deployed. Drawing distinctions within these properties seems entirely unmotivated. (Priest, 2016, pp. 83-84)

According to this objection, in order to show non- ad hocness of the restriction to $\mathrm{CP}$, it is not sufficient for nuclear Meinongianism to provide some principled way to distinguish two different kinds of properties in question. Rather, nuclear Meinongians must explain why only one of these kinds of properties can be used to characterize objects. As Priest says, the conceivability (or assumability) of any object is radically free. ${ }^{9}$ In particular, we can think about an object as having not only nuclear properties but also extranuclear

\footnotetext{
${ }^{8}$ Another main objection to nuclear Meinongianism concerns its commitment to literalism, the view that nonexistent objects actually and literally have the properties by which they are characterized. Literalism claims, for instance, that 'Sherlock Holmes is a detective' is literally true in the actual world. However, the objection goes, this is counterintuitive, since what is (intuitively) true is 'in Doyle's Holmes story, Sherlock Holmes is a detective', not 'Sherlock Holmes is a detective'. See Fine (1984) and Parsons (1980).

${ }^{9}$ For the conceivability of the impossible, see Priest (2016) and Berto (2013).
} 
properties. Priest argues that this fact shows that $\mathrm{CP}$ is applicable to both kinds of properties.

Routley disagrees with Priest. First of all, he emphasizes the distinction between the Freedom of Assumption Principle, according to which "one can assume anything, contemplate any object at all" (Routley, 1980, p. 256) and an unrestricted CP, and claims that the latter does not follow from the former. He claims:

The Freedom of Assumption Principle is unrestricted; so, it has been invalidly inferred, must the $\mathrm{CP}$ be likewise unrestricted. The argument is invalid because an item [= an object $]$ may not have all the features in terms of which it is contemplated. (Routley, 1980, p. 256)

Indeed, according to him,

any object can be assumed: by no means all of them are reliable in the way they present themselves (as $\tau x A$ ) $[\tau$ is some descriptionforming operator] as to how they are (whether they are indeed A) (Routley, 1980, p. 258)

and it is important to distinguish "what can be assumed and what reliably has the features it present itself (when assumed) as having" (Routley, 1980, p. 258). For example, consider the existent golden mountain. According to Routley, the existent golden mountain presents itself as existent, but it is not reliable with respect to this presentation. Indeed, it is not existent.

For the sake of the discussion in Section 4, it is important here to specify that the passages quoted from Routley suggests a strong connection between assumption and presentation: It suggests that $x$ presents itself as having a property $f$ iff $x$ is assumed to have $f$. Moreover, reliability seems not to be a property of object per se, but a property that an object has only relative to some description. For example, it makes sense to say that an existent golden mountain presents itself as existent, but does not make sense to say that a golden mountain presents itself as existent. However, since an existent golden mountain is a golden mountain, it is possible that they are identical.

Now recall Priest's argument for an unrestricted CP quoted above. The latter half of it appeals to the idea that we can indifferently use any property to characterize an object. Given Routley's distinction between the Freedom 
of Assumption Principle and an unrestricted CP, we can regard Priest as simply pointing out the fact that we can freely assume an object to have any properties. If Routley is correct, it doesn't follow from this that the unrestricted $\mathrm{CP}$ is true.

But, why should the application of $\mathrm{CP}$ be restricted to only characterizations consisting of nuclear properties/predicates, even though we can freely assume any object? According to Routley, there is a good reason for such a restriction and it is not an ad hoc assumption made merely to avoid contradictions that follow from naive CP. Indeed, Routley suggests that a restriction of CP and the consistency of the theory of (existent and nonexistent) objects are independent. In particular, he suggests that, even for theorists who adopt nuclear CP, it is an open matter of commonsense whether the theory of objects is consistent or paraconsistent (Routley, 1980, p. 534).

Routley provides the following argument for restricting CP. The restriction of $\mathrm{CP}$ in question is justified by the fact that it is required to exclude 'double determination' of properties. He claims:

assumptions do not, and should not settle the ontological or modal status of assumed items $\overline{\text { [= objects]: existence and possibility of }}$ items are consequential properties of items, properties which are consequential on defining features of items, but not themselves characterising features of items (Routley, 1980, p. 256)

and

The limits imposed then are intended to exclude double determination, in particular determination by description or characterisation of what is already or independently (and perhaps differently) determined (e.g. by how the world is, by other characterisation). (Routley, 1980, p. 261. My emphasis)

To make his point clear, let us call a property $p$ n-determined if whether an object has $p$ is determined by what nuclear properties it has: More precisely, for any object $o$ and the set of its nuclear properties $N$, whether $o$ has $p$ or not is uniquely fixed by $N$. Some extranuclear properties are n-determined. Completeness/incompleteness and consistency/inconsistency would be good examples of such extranuclear properties: Whether an object is complete or not is determined by which nuclear properties the object has; and whether 
an object is consistent or not is determined by which nuclear properties the object has. For these properties, such dependence is quite explicit in their definitions. For example, completeness and incompleteness could be defined as follows (cf. Parsons, 1980, p. 106)

(3) a. $x$ is complete $:=$ for any nuclear property $p, x$ has $p$ or $x$ has non- $p$ b. $x$ is incomplete $:=\sim x$ is complete

(4) a. $x$ is consistent $:=$ for any nuclear property $p, \sim(x$ has $p$ and $x$ has non- $p)$.

b. $x$ is inconsistent $:=\sim x$ is consistent

where non- $p$ is understood as the predicate negation of $p$ and $\sim$ is the classical sentential negation. Following Routley, let us assume that for any nuclear property $p$, non- $p$, its predicate negation, is also nuclear. Then, given (3a), whether an object is complete or not is uniquely determined solely by its nuclear properties. If it has $p$ or non- $p$ for any nuclear $p$, it is complete; and if not, it is not. In this way, some extranuclear properties are such that whether an object has them is determined by which nuclear properties the object has.

Now Routley's argument is explicated as follows. Consider a set of some nuclear properties $N$. Suppose that $N$ is the set of all of the nuclear properties of an object $o$, that is, $o$ has all nuclear properties in $N$ and no other nuclear properties. Since the completeness of objects are n-determined, whether $O$ is complete or incomplete is determined solely by $N$. There is no room for the additional characterization of completeness of $o$ to determine its completeness. Therefore, the property of being complete is a property which we should not use to characterize an object. Let us call this argument the double determination argument.

Routley's double determination argument is, however, problematic. First of all, not all extranuclear properties are n-determined, or, at least, their n-determinedness is contentious. Consider existence. Given Meinong's Principle of Independence, according to which Sein of an object (existence, subsistence, and non-being) is independent from its Sosein (here I understand this as its nuclear properties, or, its possession of certain nuclear properties), 
it is hardly the case that there is some set of nuclear properties whose possession by an object a priori determines its existence. ${ }^{10}$ Even the ontological argument for God's existence does not provide us an example of such a set, since, in the argument, the existence of God is inferred from its perfection, which is certainly extranuclear.

A possible reply to this objection is this: Routley proposes the following definition of existence, according to which existence is a property defined by using higher-order quantification over properties. Given this definition, even existence can be taken as n-determined.

(5) $x$ exists $:=$ for every extensional property $p$, it is necessary that, if $x$ has non- $p$, then it is not true that $x$ has $p$, and it is contingently true that, if it is not true that $x$ has $p$, then $x$ has non- $p$. (cf. Routley, 1980, p. 244; See also Paoletti, 2013, p. 283)

It is not our concern whether this definition is correct or not. For the sake of argument, let us assume that (5) is correct. Then, given this definition, it is true that a certain set of nuclear properties entails that an object which has all nuclear properties in the set does not exist: For example, if an object is red and non-red, then it doesn't exist. However, this definition is not enough to determine whether a consistent object exists or not solely in terms of its nuclear properties. In particular, merely by its nuclear properties, it is not determined whether the first conjunct in the definition (5) (= it is necessary that, if $x$ has non- $p$, then it is not true that $x$ has $p$ ') is satisfied. Therefore, even if $(5)$ is correct, it doesn't follow that existence is n-determined.

Another example is provided by intensional properties like being thought about by Russell. The round square has the property of being thought about by Russell. However, whether the intentional relation between Russell and the round square in question holds is not determined by the nuclear properties of the round square: Indeed, the round square had these nuclear properties even before Russell was born, when the intentional relation did not hold between the round square and Russell.

In this way, not all nuclear properties are n-determined, and, therefore, the double determination argument does not justify the elimination of all extranuclear properties from the class of properties to which CP is applicable.

\footnotetext{
${ }^{10}$ Even though the Principle of Independence is one of the core principles of Meinongianism and Nuclear Meinongianism adopts it, some Meinongians reject it. In particular, Priest's modal Meinongianism claims that many ordinary first order properties like being a mountain are existence-entailing: An object's having such properties entails its existence.
}

Australasian Journal of Logic (15:2) 2018 Article no. 2.1 
Secondly, if the double determination argument provides a good reason for restricting $\mathrm{CP}$, it results in the inapplicability of a certain range of characterizations consisting only of nuclear properties. To see why, let us focus on the fact that some nuclear properties are not mutually independent. The point is illustrate by a predicament which early Wittgenstein faced: A predicament concerning mutual independence of atomic propositions. In Tractatus, Wittgenstein claims that atomic propositions are mutually independent. A truth of an atomic proposition neither entails nor excludes any other atomic proposition. However, it is well-known that this claim is problematic (Anscombe, 1959). For example, the truth of the proposition that an object $o$ is blue eliminates the truth of the proposition that $o$ is red and vice versa. This mutual exclusion of these atomic propositions is naturally explained by the fact that an object's being blue excludes its being red. Now, by appealing to this mutual exclusion of color properties, one can define the negative color properties like being non-red as follows:

(6) $x$ is non-red $:=$ for some $p, p$ is a color property, $p \neq$ red, and $x$ is $p$

This definition means that an object is non-red iff it has some color property other than red. For example, if an object is blue, it is non-red. This is intuitively correct. Then, given this definition and that color properties are nuclear, non-red is extranuclear. Indeed, whether an object is non-red or not is determined solely by its nuclear properties: If some color property other than red is contained in its nuclear properties, it is non-red; if not, it isn't. Now, if this is correct, from the double determination argument, it follows that non-red should not be used to characterize an object. But, this significantly weakens the expressivity and explanatory power of nuclear Meinongianism. In particular, it renders nuclear Meinongianism incapable of treating inconsistent objects like a non-square square, at least in the way defined in (4).

In this way, Routley's double determination argument for restricting CP fails. Indeed, there is a strong argument that at least some extranuclear properties are relevant to the identity and difference between objects, and thus, should be taken as a part of objects' nature. Consider an existent golden mountain and a golden mountain. Intuitively, we can regard them to be different objects. The latter can be a nonexistent object which doesn't embody any contradiction: It may be simply nonexistent. On the other hand, the former, at least apparently, does: It is both existent and not. How can 
we distinguish them without counting existence as a part of characterization of the former?

Partly in order to accommodate this problem, Routley (1980) proposes a way of extending the range of properties to which CP is applicable, which results in a version of unrestricted $\mathrm{CP}$, in an appropriate sense. Let's see it in the next section.

\section{The supposition operator, unrestricted CP and semantics of $\xi$-terms}

Routley proposes several ways to enlarge the class of properties to which $\mathrm{CP}$ is applicable. Among them, the extension by the supposition operator is worth mentioning (Routley, 1980, pp. 270-272). Routley introduces the supposition operator (or the presentation operator), $s$, as follows:

given the predicate $f$, the operator $s$ yields the predicate $s(f)$ also written $s f$ - read 'presents itself as $f$ ' or 'says of itself that it $f$ s' or 'has suppositious $f$-ness' (Routley, 1980, p. 270)

Routley requires the following condition on $s$ :

(7) a. If $\sim \operatorname{ch}(f)$ then $\operatorname{ch}(s f)$

b. If $\operatorname{ch}(f)$ then $s f \approx f$

where 'ch' is a primitive predicate for characterizing predicates (that is, $\operatorname{ch}(f)$ means that $f$ is a characterizing, and thus, nuclear property) and $\approx$ is Leibnitz identity. ${ }^{11}$

To see his extension of $\mathrm{CP}$, let us now introduce his $\xi$ operator. $\xi$ is a description-forming operator. ' $\xi x A(x)$ ' roughly corresponds to the English indefinite NP 'an $A$ '. Then, according to Routley, (i) $\xi x A(x)$ is an object which presents itself as $A$. However, (ii) this presentation may not be reliable and it may not be the case that $A(\xi x A(x))$. (ii) is reflected in the restricted

\footnotetext{
${ }^{11}$ Leibnitz identity is identity whose behavior is fixed by its reflexivity and substitutivity:

$(8) \approx 1 . u \approx u$

$\approx 2 . u \approx v \supset . A(u) \supset A(v)$ (Routley, 1980, p. 200)
}

Australasian Journal of Logic (15:2) 2018 Article no. 2.1 
CP. However, how about (i)? Routley commits himself to the idea that it must always be the case that $\xi x A(x)$ presents itself as having $A$. This idea is reflected in the following $\mathrm{CP}$, which is formulated by using the supposition operator.

(9) JCP: $s A(\xi x A)$ for every $A$ for which $s A$ is well-defined. (Routley, 1980, p. 270)

Four remarks on JCP. First of all, JCP can be regarded as a version of unrestricted $\mathrm{CP}$ in the sense that it can apply to any characterization consisting of nuclear properties and/or extranuclear properties. This is because the supposition operator $s$ is applicable to all nuclear properties and all extranuclear properties.

Secondly, from JCP and Routley's definition of the supposition operator, we can infer nuclear CP. Suppose that $A$ is a characterization consisting only of nuclear properties. Then, since $s A$ is the same as $A$, therefore, $A(\xi x A(x))$ holds.

Thirdly, according to Routley, JCP enables us to distinguish an existent golden mountain from a golden mountain (cf. Routley, 1980, p. 270). The former is an object which has the nuclear properties of being golden, being a mountain and s(existent), but the latter is an object which has being golden, being a mountain and does not have s(existent). We will be back to this point soon and see it in more detail from a semantic viewpoint.

Fourthly and finally, Routley applies the supposition operator to his theory of fictional objects. It is often the case that a fictional story (explicitly or implicitly) describes a character as having not only certain nuclear properties but also certain extranuclear properties. For example, it is natural to hold that Holmes is described not only as being a detective but also as being existent in Doyle's Holmes stories. According to Routley, "[t]he full object $\mathrm{d}$ is the object that has all the features precisely that [a story] $\overline{\mathrm{S} \text { ascribes }}$ to $\mathrm{d}$... The full object has ... all characterising features ... [and] all noncharacterising features ... ascribed to it in S in s-form" (Routley, 1980, pp. 596-597). For example, Holmes has the nuclear properties of being a detective and of presenting itself as existent (s(existent)), but lacks the extranuclear property of being existent.

Given, in particular, the third and forth remarks, even though Routley does not explicitly specify this, it is reasonable to suppose that JCP goes along with the following semantics of $\xi$-terms.

Australasian Journal of Logic (15:2) 2018 Article no. 2.1 
(10) ' $\xi x A(x)$ ' denotes an arbitrary object which has $s A$, that is,

$$
\llbracket \xi x A(x) \rrbracket=c(\llbracket s A \rrbracket)
$$

where $c$ is a choice function from the power set of the domain, $D$, to $D$ such that $c\left(D^{\prime}\right) \in D^{\prime}$ for any non empty $D^{\prime}(\subseteq D)$.

Consider ' $\xi x$ ( $x$ is an existent golden mountain)'. ${ }^{12}$ As Routley does, let us suppose that the supposition operator $s$ has the following distributive property: $s(A \& B) \approx(s A \& s B)$ (cf. Routley, 1980, p. 270). Moreover, let us recall that, if $f$ is nuclear, then $s f$ is the same as $f$. Then, according to (10), ' $\xi x(x$ is an existent golden mountain)' denotes an arbitrary object which has the nuclear properties of being golden, being a mountain, and presenting itself as existent. This object does not have the extranuclear property of being existent, since, as a matter of (extra-logical) fact, there exist no golden mountains.

The semantics of $\xi$-terms specified in (10), together with JCP, will solve the problem concerning the distinction between an existent golden mountain and a golden mountain. Originally, Routley's semantics of $\xi$-terms is like that of Hilbert's $\varepsilon$-terms, except that the former lacks existential commitment (Routley, 1980, pp. 197-199). ' $\xi x A(x)$ ' denotes an arbitrary (existent or nonexistent) object which satisfies $A(x)$, if something satisfies $A(x)$. A problem arises when no object satisfies $A$. For example, even for Meinongians, it is hard to reject that no object satisfies (in the actual world) the condition $x$ is golden \& $x$ is a mountain \& $x$ is existent, which consists of two nuclear properties and an extranuclear properties. Then, what does ' $\xi x(x$ is golden \& $x$ is a mountain \& $x$ is existent $)$ ', in short, ' $\xi x(x$ is an existent golden mountain)' denote? An option is, reflecting Hilbert's semantics, that when no object satisfies $A(x)$, then ' $\xi x A(x)$ ' denotes an arbitrary object taken from the whole domain. Then, ' $\xi x(x$ is an existent golden mountain)' denotes an arbitrary object which may not even be a mountain. At some point, Routley gives us a less radical solution. According to him, when $A$ contains both nuclear properties and extranuclear properties, then ' $\xi x A(x)$ ' denotes an arbitrary object which satisfies all of nuclear properties specified by $A$. According to this semantics, ' $\xi x$ ( $x$ is an existent golden mountain)'

\footnotetext{
${ }^{12} \mathrm{I}$ assume that $x$ is an existent golden mountain $:=($ being existent \& being golden \& being a mountain $)(x)$.
}

Australasian Journal of Logic (15:2) 2018 Article no. 2.1 
denotes an arbitrary nonexistent object which is a golden mountain. But, if this is correct, there is no semantic difference between ' $\xi x(x$ is an existent golden mountain)' and ' $\xi x(x$ is a golden mountain)', which, intuitively speaking, have different semantic behaviors. The semantics in (10), which seems to be implicitly assumed by Routley, enables us to make the required distinction. Indeed, in this semantics, ' $\xi x$ ( $x$ is an existent golden mountain)' denotes an arbitrary object which has the nuclear properties of being golden, being a mountain, and presenting itself as existent, and ' $\xi x(x$ is a golden mountain)' denotes an arbitrary object which has the nuclear properties of being golden and being a mountain, but may not have the nuclear property of presenting itself as existent. ${ }^{13}$

So far I have given an exposition of Routley's JCP and the supposition operator $s$, and examined how to modify semantics of $\xi$-term by incorporating $s$. In the next section, I propose a possible revision of JCP through interpreting the supposition operator $s$ as one which creates the intensional predicate $s(f)$ read as 'being assumed to have $f$ '.

\section{Beyond Routley's nuclear Meiongianism}

As we have seen in Section 2, Routley suggests that there is a strong connection between presentation and assumption: $x$ presents itself as having a property $f$ iff $x$ is assumed to have $f$. This connection is further supported by his comment that $s(f)$ is read not only as presenting itself as $f$ but also as having suppositious $f$-ness (Routley, 1980, p. 270). Indeed, the latter paraphrase strongly suggests that $s(f)$ is the property of being supposed to have $f$ or one of being assumed to have $f$. Moreover, this reading explains why JCP holds. As we have seen, Routley agrees with Priest that we can freely assume any object to have any (nuclear or extranuclear) property. We can take JCP which says that $\xi x A$ presents itself as satisfying $A$ as just a paraphrase of the Freedom of Assumption Principle.

\footnotetext{
${ }^{13}$ However, unfortunately, it is easy to point out a problem that is not yet solved by the semantics in (10). For example, it fails to distinguish an existent golden mountain and a golden mountain presenting itself as existent. This is because, according to (10), there is no semantic difference between $\xi x$ ( $x$ is an existent golden mountain)' and ' $\xi x$ ( $x$ is a golden mountain presenting itself as existent)': Both of them denote an arbitrary object which has the nuclear properties of being golden, being a mountain, and presenting itself as existent.
}

Australasian Journal of Logic (15:2) 2018 Article no. 2.1 
So, it is reasonable to interpret $s(f)$, the property of presenting itself as having $f$, as the property of being assumed to have $f$. However, it is also the case that this understanding of $s(f)$ has a certain conflict with, first, what Routley explicitly says and, second, his own theory. Firstly, he says that "[t]he "suppositious" terminology, adopted from Meinong, is thus somewhat misleading, since $s f$ is presumably extensional" (Routley, 1980, p. 270). Secondly, as we have seen, (a) intensional properties like being thought about by Russell are extranuclear; (b) the property of being assumed to have $f$ is naturally taken as an intensional property; and (c) $s(f)$ is nuclear, for any $f$. (a), (b) and (c) are incompatible, once we understand $s(f)$ as the property of being assumed to have $f$.

In this way, what Routley says about the nature of the supposition operator is ambivalent. On the one hand, $s$ is an operator which, combined with any property $f$, creates the intensional property of being assumed to have $f$. On the other hand, $s(f)$ is not an intensional property, since, it is a nuclear property to which we can apply $\mathrm{CP}$ and no intensional property is nuclear. Routley officially endorses the latter claim. However, as far as I can see, this leaves it a mystery what exactly it means to say that an object presents itself as having a property. Moreover, it is not clear why the supposition operator has the features specified by Routley: How can the supposition operator turn an extranuclear property into a nuclear property? Why does not adding it to a nuclear property make any change to its nuclear status? All these things are merely assumed and left unexplained. They are just ad hoc assumptions made to incorporate an operator with the desired features into the framework of nuclear Meinongianism. ${ }^{14}$ On the other hand, once we understand $s(f)$ as an intensional property, as we will see soon, we can give a certain explanation to it based on world semantics.

So, pace Routley, let us stick with our interpretation of $s(f)$ as the intensional property of being assumed to have $f$. If so, we need to abandon one among (a), (b) and (c). The most reasonable choice is to abandon (c). Indeed, it is not clear why merely suppositious $f$-ness constitutes the nature of an object. However, abandoning (c) results in some substantial changes in Routley's original theory. First of all, we cannot maintain the equation of $f$ and $s(f)$ for nuclear $f$, since $f$ is nuclear but $s(f)$ isn't. Neither does $s$ convert an extranuclear property into a nuclear property. Two defining

\footnotetext{
${ }^{14}$ See Berto (2013, p. 128) for a similar argument against the so-called watering-down principle.
}

Australasian Journal of Logic (15:2) 2018 Article no. 2.1 
features which Routley specifies for the supposition operator are no longer maintained. More importantly, we can no longer regard JCP as a version of nuclear $\mathrm{CP}$, since JCP allows us to use $s(f)$, which is extranuclear, to characterize an object. So, at this point, our investigation goes beyond Routley's nuclear Meiongianism. However, it is still the case that our interpretation of the supposition operator is on the same track as Routley's in the sense that, as we will see, under our interpretation, JCP holds.

In what follows, I give a sketch of a world semantics of the supposition operator $s$ based on the understanding of $s(f)$ as the property of being assumed to have $f$.

Let $\varphi$ be any first-order one-place predicate. $\varphi$ may be atomic, or may be complex. The supposition operator $s$ is an operator which creates an intensional predicate $s(\varphi)$ for any $\varphi \cdot s(\varphi)$ is read as 'being assumed to have $\varphi$ '. A world semantics of $s(\varphi)$ is given based on the idea that $x$ 's being assumed to have $\varphi$ is analyzed as $x$ 's having $\varphi$ in all worlds where everything assumed is realized.

A model $<W, D, R^{s}, C, \llbracket \rrbracket>$ is defined as follows. $W$ is a set of worlds. $D$ is a set of individuals (as the constant domain). $R^{s}$ is a relation between worlds such that $w R^{s} w^{\prime}$ iff $w^{\prime}$ is a world where everything assumed in $w$ is realized. I require that $R^{s}$ satisfy the following condition:

(11) For any $w$ there must be some $w^{\prime}$ such that $w R^{s} w^{\prime}$

This requirement, together with (14), which is introduced below, ensures that any assumption is realized at some world. $C$ is a set of choice functions (see $(10))$. 『】 is an interpretation function for nonlogical primitive constants.

$\llbracket \rrbracket$ is expanded for $s(\varphi)$ and $\xi$-terms as follows. For $s(\varphi)$ :

$$
\llbracket s(\varphi) \rrbracket^{w, g}=\left\{d \in D \mid \text { for any } w^{\prime}, \text { if } w R^{s} w^{\prime}, \text { then } d \in \llbracket \varphi \rrbracket^{w^{\prime}, g}\right\}
$$

where $g$ is a variable assignment. Informally, an object $d$ has the property $s(\varphi)$ in the world $w$ iff $d$ has $\varphi$ in all worlds where what is assumed in $w$ holds.

The semantics of $\xi$-terms in (10) is elaborated as follows. Let us first suppose that $\xi x \varphi(x)$ is defined only if $\varphi$ is a one place predicate and $x$ is not free in $\varphi$. Then, the interpretation of ' $\xi x \varphi(x)$ ' relative to a choice function $c$ is defined as follows.

$$
\llbracket \xi x \varphi(x) \rrbracket^{w, g, c}=c\left(\llbracket s(\varphi) \rrbracket^{w, g}\right)
$$

Australasian Journal of Logic (15:2) 2018 Article no. 2.1 
Informally, ' $\xi x \varphi(x)$ ' denotes an arbitrary object which is assumed to have $\varphi$.

It is important to specify that the well-definedness of (13) depends on the non-emptiness of $\llbracket s(\varphi) \rrbracket^{w, g}$, since $c$ is defined only for nonempty subsets of $D$. In order to ensure the non-emptiness of $\llbracket s(\varphi) \rrbracket^{w, g}$, I require that a model satisfy the following condition:

(14) For any $w$ and $\varphi$, some object $d$ is such that for any $w^{\prime}$, if $w R^{s} w^{\prime}$, then $d \in \llbracket \varphi \rrbracket^{w^{\prime}, g}$.

(14), together with (11), ensures that any assumption is realized at some world. Note that this doesn't mean that any assumption is realized at the actual world ( $R^{s}$ needs not be reflexive, and thus @ $R^{s} @$ needs not hold). We can take this as reflecting Routley's distinction between the Freedom of Assumption Principle (any assumption about any object is realized at some world) and the unrestricted CP (any assumption about any object holds in the actual world). Of course, some assumption is true and realized at the actual world. In this case, ' $\xi x \varphi(x)$ ' denotes an arbitrary object which is not only assumed to have $\varphi$ but also actually has $\varphi$.

The truth condition of atomic sentences containing $\xi$-term is defined as follows (for simplicity, I treat only cases where a $\xi$-term is combined with only a one-place predicate).

$$
\llbracket \psi(\xi x \varphi(x)) \rrbracket^{w, g}=1 \text { iff for some } c \in C, \llbracket \xi x \varphi(x) \rrbracket^{w, g, c} \in \llbracket \psi \rrbracket^{w, g}
$$

Given these interpretations, it is quite easy to see that JCP holds.

(16) For any $\varphi$ for which $\xi x \varphi(x)$ is defined, for any $w$ and for any $g$, $\llbracket s(\varphi)(\xi x \varphi(x)) \rrbracket^{w, g}=1$

proof: By definition, $\llbracket s(\varphi)(\xi x \varphi(x)) \rrbracket^{w, g}=1$ iff

for some $c \in C, \llbracket \xi x \varphi(x) \rrbracket^{w, g, c} \in \llbracket s(\varphi) \rrbracket^{w, g}$ iff

for some $c \in C, c\left(\llbracket s(\varphi) \rrbracket^{w, g}\right) \in \llbracket s(\varphi) \rrbracket^{w, g}$

The last one holds, since, for any $c$ and any non-empty $D^{\prime}(\subseteq D), c\left(D^{\prime}\right) \in D^{\prime}$ and, by (11) and (14), $\llbracket s(\varphi) \rrbracket^{w, g}$ is not empty.

So far this has been a sketch of a formal treatment of the supposition operator interpreted as an intensional-predicate-forming operator. Many things are left unspecified. Among them, the following is worthwhile mentioning. At the beginning, it is said that $\varphi$ may be complex. Nothing is said about which 
complex predicates the current theory has; nor is about how they semantically behave. Indeed, depending on how syntax and semantics of complex predicates are elaborated, we obtain different theories. For example, suppose that we have complex predicates of the form $\lambda x . \neg A(x)$ and give them semantics which ensures the biconditional $\lambda x . \neg A(x)(y)$ iff $\neg A(y)$. Further suppose that we have complex predicates of the form $\lambda x .(A(x) \wedge B(x))$ and give them semantics which ensures the biconditional $\lambda x .(A(x) \wedge B(x))(y)$ iff $A(y) \wedge B(y)$. Then, the above framework, in particular due to (11) and (14), requires impossible worlds where the contradiction $A(x) \wedge \neg A(x)$ holds. Moreover, an extreme theory is obtained if we specify syntax and semantics of complex predicates as follows: For any formula $A$ containing at least one free occurrence of $x, \lambda x . A$ is a one-place predicate; and for any $A, \lambda x . A(y)$ iff $A[x / y]$. From this syntax and semantics of complex predicates, it follows that some world in a model is trivial: From (11) and (14), for any $B$, in some world $w$ something is in $\llbracket \lambda x .(x \wedge B) \rrbracket^{w, g}$. In such a world, given the semantics for complex predicates, $b \wedge B$ holds for some $b$, and thus, $B$ does too. It is worthwhile mentioning that the resulting theory is a version of modal Meinongianism proposed and defended by Priest (2005, 2016). Modal Meinongianism endorses the following modal CP, which is applicable to any characterization without any restriction.

(17) modal CP: For any characterization, some object satisfies it in some (possible or impossible) world(s) (and it may not be the actual world.)

Indeed, in our semantics, the combination of (11) and (14), together with the syntax and semantics of complex predicates specified at the end of the last paragraph, is regarded as a version of modal CP. ${ }^{15}$

\footnotetext{
${ }^{15} \mathrm{It}$ is worth mentioning that the semantics presented here is only one way of interpreting the supposition operator, which would be open to different interpretations. In particular, given the fact that an assumption/supposition as a cognitive act is made by an agent, it would be natural and reasonable to relativize the supposition operator to agents (and maybe other contextual factors like time points). A way to incorporate this relativity to a theory of the supposition operator is to introduce agent-indexed supposition operators of the form $s_{a}$, read as 'being assumed to have by $a$ ' (as an intentional operator $\Phi$ with a term $a$ in Priest $(2005,2016), a \Phi$ combines with any formula to form a formula $a \Phi A$, read as ' $a \Phi$ s that $A$ '. ' $a \Phi A$ ' corresponds to a propositional attitude reports like 'John believes that Kyoto is beautiful' and so on). A model contains, instead of $R^{s}$, a set of accessibility relations on $W, \mathcal{R}$, such that for each agent $d, R_{d}^{s} \in \mathcal{R}$, where $w R_{d}^{s} w^{\prime}$ iff $w^{\prime}$ is a world where everything assumed by $d$ in $w$ is realized (cf. Priest, 2005, 2016, chap. 1).
}

Australasian Journal of Logic (15:2) 2018 Article no. 2.1 
The interpretation of ' $\xi x \varphi(x)$ ' requires careful treatments. There are at least two different ways of defining it, which leads to two slightly different semantics, respectively.

The first option is $\left(13^{\prime}\right)$ :

(13') $\llbracket \xi x \varphi(x) \rrbracket^{w, g, c}=c\left(\left\{d \in D \mid\right.\right.$ for some agent $e$, for any $w^{\prime}$, if $w R_{e}^{s} w^{\prime}$, then $\left.\left.d \in \llbracket \varphi \rrbracket^{w^{\prime}, g}\right\}\right)$.

According to (13'), ' $\xi x \varphi(x)^{\prime}$ ' denotes an arbitrary object such that someone assumes it to have $\varphi$. The interpretation of ' $s_{a}(\varphi)$ ' is defined as follows:

$\left(12^{\prime}\right) \llbracket s_{a}(\varphi) \rrbracket^{w, g}=\left\{d \in D \mid\right.$ for any $w^{\prime}$, if $w R_{\llbracket a \rrbracket w, g}^{s} w^{\prime}$, then $\left.d \in \llbracket \varphi \rrbracket^{w^{\prime}, g}\right\}$,

and, the requirement (14) is modified as follows:

(14') For any $w$ and $\varphi$, some object $d$ and some agent $e$ are such that for any $w^{\prime}$, if $w R_{e}^{s} w^{\prime}$, then $d \in \llbracket \varphi \rrbracket^{w^{\prime}, g}$.

From these definitions and requirement the following version of JCP is derived.

(16') For any $\varphi$ for which $\xi x \varphi(x)$ is defined, for any $w$, there is an agent $e$ such that for any $g$ which assigns $e$ to $x, \llbracket s_{x}(\varphi)(\xi x \varphi(x)) \rrbracket^{w, g}=1$

The second option is to define a context-sensitive semantics of $\xi$-terms. Suppose that $\langle w, e\rangle$, a pair of a world and an agent, is a context whose agent of assumption is $e$. Then, the interpretation of ' $\xi x \varphi(x)$ ' relative to a choice function $c$ in a context $\langle w, e\rangle$ is defined as follows:

(13") $\llbracket \xi x \varphi(x) \rrbracket^{\langle w, e\rangle, g, c}=c\left(\left\{d \in D \mid\right.\right.$ for any $w^{\prime}$, if $w R_{e}^{s} w^{\prime}$, then $\left.\left.d \in \llbracket \varphi \rrbracket^{\left\langle w^{\prime}, e\right\rangle, g}\right\}\right)$

According to (13"), ' $\xi x \varphi(x)$ ' denotes an arbitrary object such that the agent of the context in question assumes it to have $\varphi$. Let us suppose that only $\xi$-terms are context-sensitive in the relevant sense: So, any atomic expression $\varepsilon$, $\llbracket \varepsilon \rrbracket^{\langle w, e\rangle, g}=\llbracket \varepsilon \rrbracket^{w, g}$. The interpretation of ' $s_{a}(\varphi)$ ' is defined as follows:

(12") $\llbracket s_{a}(\varphi) \rrbracket^{\langle w, e\rangle, g}=\left\{d \in D \mid\right.$ for any $w^{\prime}$, if $w R_{\llbracket a \rrbracket w, g}^{s} w^{\prime}$, then $\left.d \in \llbracket \varphi \rrbracket^{\left\langle w^{\prime}, e\right\rangle, g}\right\}$

and the truth condition of atomic sentences containing a $\xi$-term in a context is:

$$
\llbracket \psi(\xi x \varphi(x)) \rrbracket^{\langle w, e\rangle, g}=1 \text { iff for some } c \in C, \llbracket \xi x \varphi(x) \rrbracket^{\langle w, e\rangle, g, c} \in \llbracket \psi \rrbracket^{\langle w, e\rangle, g}
$$

(14) is modified as follows:

(14") For any $w$ and $\varphi$, some object $d$ and some agent $e$ are such that for any $w^{\prime}$, if $w R_{e}^{s} w^{\prime}$, then $d \in \llbracket \varphi \rrbracket^{\left\langle w^{\prime}, e\right\rangle, g}$.

In this semantics, the following version of JCP holds.

(16") For any $\varphi$ for which $\xi x \varphi(x)$ is defined, for any $w$, there is an agent $e$ such that for any $g$ which assigns $e$ to $x, \llbracket s_{x}(\varphi)(\xi x \varphi(x)) \rrbracket^{\langle w, e\rangle, g}=1$

Australasian Journal of Logic (15:2) 2018 Article no. 2.1 


\section{Conclusion}

We have arrived at the following picture of Routley's Meinongianism: On the one hand, based on the distinction between an unrestricted CP and the Freedom of Assumption Principle, Routley endorses a restricted CP. Even though his double determination argument fails to give a good reason to restrict the applicability of CP to nuclear properties, it is still the case that the distinction is quite reasonable: We can freely assume any object to have any property, but this doesn't mean that the assumed object really has the assumed property. Assumptions are unrestricted, but may not be realized. On the other hand, he proposes a different version of CP, JCP, which does justice to the Freedom of Assumption Principle. According to JCP, for any $A, \xi x A$ presents itself as having $A$. Routley formulates JCP by using the supposition operator, $s$, which creates a property of presenting itself as having $f$. Assuming $s f$ is nuclear no matter whether $f$ is nuclear or extranuclear, he claims that JCP is still formulated within the framework of nuclear Meinongianism. However, this assumption is ad hoc and it is natural to interpret the supposition operator $s$ as an intensional-predicate-forming operator. Based on this interpretation, I diverged from Routley's original nuclear Meinongianism and gave a formal treatment of the supposition operator within the framework of world semantics, which assimilates his view with modal Meinongianism. In this reconstruction of Routley's Meinongianism, his endorsement of a restricted CP, on the one hand, and the Freedom of Assumption Principle, on the other hand, is recaptured in the following way: some assumptions of an object fail to be the case in the actual world; however, any assumption of any object is realized in some (possible or impossible) world(s).

Finally, it is worthwhile mentioning that, later, Routley/Sylvan explicitly endorsed such twofold, pluralist metaphysics. ${ }^{16}$ By using a metaphor of the city center and suburbs, Sylvan (1995) proposes that only the restricted CP holds in the city center (that is, the actual world ${ }^{17}$ ), but any characterization is realized in suburbs (that is, some impossible worlds) and, thus, CP is unrestricted there. As this paper has shown, we can find a sign of this pluralist picture even in his early version of Meinongianism.

\footnotetext{
${ }^{16}$ I thank Filippo Casati for indicating me the similarity between my reconstruction of Routley (1980) and the late Routley/Sylvan's thought.

${ }^{17}$ More precisely, the actual world $s$, since, surprisingly, Sylvan endorses the plurality of the actual worlds. See Sylvan (1997).
}

Australasian Journal of Logic (15:2) 2018 Article no. 2.1 


\section{Acknowledments}

I would like to thank Filippo Casati and the anonymous reviewers for their helpful and constructive comments and suggestions.

\section{References}

[1] Anscombe, G. E. M. (1959). An Introduction to Wittgenstein's Tractatus, Second Edeition, Revised, New York: Harper and Row Publishers.

[2] Berto, F. (2013). Existence as A Real Property: The Ontology of Meinongianism, London: Springer.

[3] Berto, F. and M. Plebani (2015). Ontology and Metaontology: A contemporary Guide, London: Bloomsbury Academic.

[4] Findlay, J. N., (1963). Meinong's Theory of Objects and Values, Second Edition, Oxford: Clarendon Press.

[5] Fine, K. (1982). 'The Problem of Non-Existents I: Internalism', Topoi, 1: $97-140$.

[6] Jacquette, D. (2015). Alexius Meaning: the Shepherd of Non-Being, (Synthese Library, vol 360), Springer.

[7] Paoletti, M. (2013), 'Commentary on Exploring Meiong's Jungle and Beyond: an Investigation of Noneism and the Theory of Items', Humana. Mente: Journal of Philosophical Studies, 25. 275-292.

[8] Parsons, T. (1980). Nonexistent Objects, New Haven: Yale University Press.

[9] Priest, G. (2005). Towards Non-Being: The Logic and Metaphysics of Intentionality, Oxford: Oxford University Press.

[10] Priest, G. (2016). Towards Non-Being: The Logic and Metaphysics of Intentionality, the 2nd Edition, Oxford: Oxford University Press.

[11] Rapaport, W. (1984). 'Review of Exploring Meinong's Jungle and Beyond', Philosophy and Phenomenological Research, 44(4), 539-552.

Australasian Journal of Logic (15:2) 2018 Article no. 2.1 
[12] Routley, R. (1980). Exploring Meinong's Jungle and Beyond, Canberra: RSSS, Australian National University.

[13] Russell, B. (1905). 'On Denoting', Mind, 14, No. 56, pp. 479-493.

[14] Sylvan, R. (1995). 'Re-exploring Item-Theory', Grazer Philosophische Studien, 50, 47-85.

[15] Sylvan, R. (1997). Transcendental Metaphysics: From Radical to Deep Pluralism, Isle of Harris: White Horse Press. 